\title{
Propaganda Eleitoral Antecipada: Uma Análise de Postagens em Mídias Sociais
}

\author{
Márcio Silva $^{1,2}$, Samuel Guimarães ${ }^{1}$, Josemar Caetano ${ }^{1}$, Marcelo Araújo ${ }^{1}$, \\ Jonatas Santos ${ }^{1}$, Julio C. S. Reis ${ }^{3}$, Ana Silva ${ }^{1}$, Fabrício Benevenuto ${ }^{1}$, Jussara Almeida ${ }^{1}$ \\ ${ }^{1}$ Universidade Federal de Minas Gerais (UFMG) - Brasil \\ ${ }^{2}$ Universidade Federal de Mato Grosso do Sul (UFMS) - Brasil \\ ${ }^{3}$ Universidade Federal de Viçosa (UFV) - Brasil \\ marcio@facom.ufms.br, \{samuelsg,mmra, jonatashds\}@ufmg.br, jreis@ufv.br \\ \{josemarcaetano, ana.coutosilva, fabricio, jussara\}edcc.ufmg.br
}

\begin{abstract}
The popularization of the use of online social networks as platforms for political debate has brought new challenges like the improper propagation of electoral advertisements. On the one hand, voters use social networks to interact, inform themselves, and get to know their candidates. On the other hand, true digital podiums have emerged for candidates to spread their ideas, fight opponents and ask for votes. Thus, pre-candidates can use the platforms to request votes outside the electoral period, a practice known as early electoral propaganda. Although legislation on this exists, the lack of digital tools and detection methods for this practice can be exploited. In this context, this work presents a methodology to help the detection of this type of propaganda. We collect and characterize data from Twitter and Facebook during three pre-election periods in Brazil (2016, 2018, and 2020), presenting challenges and important finds about the use of social networks to create these ads.
\end{abstract}

Resumo. A popularização do uso de redes sociais online como plataformas para o debate político trouxe novos desafios como a propagação indevida de propagandas eleitorais. Por um lado, os eleitores usam as redes sociais para interagir, buscar informações e conhecer seus candidatos. Por outro lado, surgiram verdadeiros palanques digitais para candidatos difundirem suas ideias, atacar adversários e pedir votos. Dessa forma, pré-candidatos podem usar as plataformas para pedir voto fora do periodo eleitoral, prática conhecida como propaganda eleitoral antecipada. Apesar de existir legislação sobre isso, a falta de ferramentas digitais e métodos de detecção dessa prática pode ser explorada. Neste contexto, este trabalho apresenta uma metodologia para auxiliar a detecção desse tipo de propaganda. Nós coletamos e caracterizamos dados do Twitter e Facebook durante três períodos eleitorais brasileiros (2016, 2018 e 2020), e apresentamos desafios e descobertas importantes sobre o uso das redes sociais para realização dessas propagandas.

\section{Introdução}

As redes sociais online se tornaram atualmente uma imprescindível ferramenta de comunicação entre eleitores e seus candidatos. Porém, esta comunicação pode ser deturpada quando perfis online de candidatos se tornam verdadeiros palanques em períodos 
eleitorais. Embora permitido, as eleições possuem regras claras a respeito do uso correto destas plataformas como ferramentas da campanha para equilibrar a competição entre os candidatos. Por outro lado, surge a preocupação do uso indevido das redes sociais em períodos pré-eleitorais para realização de propagandas políticas, prática vedada pela legislação eleitoral vigente (Lei Eleitoral n 13.488, 6 de outubro, 2017).

Antes do surgimento das redes sociais, as campanhas eleitorais eram realizadas integralmente por meio do horário político gratuito ou presencialmente junto ao eleitorado. Neste cenário, a fiscalização e garantia de cumprimento da lei eleitoral na utilização de meios de comunicação em massa eram mais simplificadas, sendo apenas necessário monitorar o que era veiculado em canais de rádio e televisão. Porém, as plataformas de redes sociais forneceram uma nova forma de comunicação entre os eleitores e candidatos com seu eleitorado.

Estudos apontam que houve interferência externa nas eleições americanas em 2016 através de anúncios [Ribeiro et al. 2019]. Com o receio de que algo semelhante pudesse acontecer nas eleições brasileiras, em 2017, a lei eleitoral trouxe modernizações em suas regras por meio da regulamentação do uso das redes sociais para campanhas eleitorais. Porém, o uso indevido de campanhas eleitorais nestas plataformas não se restringe ao período oficial de campanha e, quando ocorre antes do período permitido, caracteriza-se a chamada propaganda eleitoral antecipada. Diante deste cenário, esta pesquisa consolidase em um primeiro esforço que visa avaliar dados coletados de Twitter e Facebook nos períodos pré-eleitorais de 2016, 2018 e 2020 com o objetivo de explorar a propaganda eleitoral antecipada.

O restante deste artigo está organizado como segue. A Seção 2 discute trabalhos relacionados, enquanto a Seção 3 apresenta como os conjuntos de dados explorados neste trabalho. Os resultados da caracterização das narrativas presentes na publicações coletadas são apresentados e discutidos na Seção 4. Por fim, a Seção 5 conclui este trabalho e apresenta possíveis trabalhos futuros.

\section{Trabalhos Relacionados}

Entre as pesquisas que buscam detectar o discurso político, encontramos principalmente dois grupos: (i) o primeiro focado em modelos de aprendizado de máquina e rotulação manual e; (ii) o segundo que utiliza léxicos ou conjuntos de palavras-chave. Como exemplo do primeiro grupo, os autores de [Silva et al. 2020] e [Oliveira et al. 2020] construíram modelos de classificação com o objetivo de identificar conteúdo político no Facebook e Twitter, respectivamente. Por outro lado, os autores de [Conover et al. 2011] e [Grimaldi 2019] utilizaram uma lista de hashtags para identificar tweets caracterizados como comunicação ideológica, visando analisar como as mídias sociais moldam a opinião pública e permitem a comunicação entre grupos políticos distintos. Nossa estratégia se relaciona principalmente ao segundo grupo de artigos, tendo gerado uma lista de termos relacionados à propaganda antecipada usada na busca por tweets e postagens no Facebook. Além da rotulação de dados que poderão ser utilizados por outros trabalhos.

\section{Base de dados}

Nossa base de dados é composta por tweets da base histórica do Twitter. Ao contrário da API de pesquisa que realiza buscas em uma amostra de tweets publicados nos últimos 7 
dias, a API histórica fornece acesso a postagens realizadas desde $2006,{ }^{1}$ definido como TWITTER-DATASET. Nós também coletamos posts do Facebook diretamente através da ferramenta CrowdTangle. ${ }^{2}$ Essa ferramenta de relatórios coleta informações sobre postagens realizadas no Facebook, Instagram, Reddit e Twitter, definido como FACEBOOKDATASET.

\subsection{Coleta}

A coleta de dados nas duas redes sociais foi realizada levando em conta os períodos préeleitorais compreendidos entre 1 de janeiro de 2016 a 15 de agosto de 2016, 1 de janeiro de 2018 a 15 de agosto de 2018 e 1 de janeiro de 2020 a 25 de setembro de 2020, respectivamente.

Inicialmente, nós elencamos 54 palavras-chave para a coleta na base histórica do Twitter e no CrowdTangle. Estas palavras-chave foram sugeridas por especialistas de instituições fiscalizadoras das eleições brasileiras. Em resumo, são palavras-chave que indicam o pedido de voto explícito ao eleitor, ou pedidos de voto implícitos (ex.: “...conto com você nestas eleições."). Por questões de clareza, apresentamos na Tabela 1 um quadro consolidado com a quantidade de publicações retornadas pelas 5 palavras-chave mais comuns nas publicações em cada plataforma.

Tabela 1. Top 5 palavras-chave com mais publicações (Twitter e Facebook) utilizadas na coleta (2016, 2018 e 2020).

\begin{tabular}{|l|r|l|r|}
\hline Palavras-chave (Twitter) & \# Tweets & Palavras-chave (Facebook) & \# Posts \\
\hline \hline vote em quem & 35.388 & juntos nessa caminhada & 2.377 \\
\hline vote em mim & 789 & votar em candidato & 1.768 \\
\hline vote no candidato & 750 & vote em quem & 1.515 \\
\hline votar em mim & 732 & vote em candidato & 1.482 \\
\hline vote em candidato & 607 & votar em candidatos & 1.441 \\
\hline
\end{tabular}

No Twitter, coletamos no total 3.244 postagens realizadas por 2.268 usuários diferentes em 2016, 9.744 postagens de 8.860 usuários em 2018 e 38.296 postagens de 29.180 usuários em 2020. É importante salientar que duas ou mais palavras-chave podem ser encontradas em um mesmo tweet. No Crowdtangle, coletamos 13.937 publicações no total, sendo 1.949 de 2016, 3.230 de 2018 e 8.758 em 2020, de um número desconhecido de usuários anonimizados. Após a coleta, observamos que as palavras-chave que nos permitem encontrar prováveis propagandas eleitorais, também são usadas por conteúdo não político como votações online. Para resolver isso, criamos uma blacklist ${ }^{3}$ de termos com o propósito de que não fossem retornamos esses tipos de conteúdo.

\section{Caracterização dos Dados}

Primeiramente, nós realizamos uma caracterização geral dos dados coletados. Nossa primeira análise consistiu em examinarmos a distribuição temporal das publicações em ambos os conjuntos de dados. A Figura 1 apresenta o número de tweets postados semanalmente em cada período pré-eleitoral analisado. De imediato, observamos um intenso

\footnotetext{
${ }^{1}$ https://developer.twitter.com/en/docs/twitter-api/search-overview

${ }^{2}$ http://crowdtangle.com/

${ }^{3}$ https : / / facom.ufms.br/ marcio/brasnam2 021/blacklist.txt
} 
fluxo de postagens durante o primeiro mês de cada ano. Uma análise dos tweets nos revelou que, para 2016, este fluxo teve grande relação com a pré-candidatura de Donald Trump à presidência dos Estados Unidos.

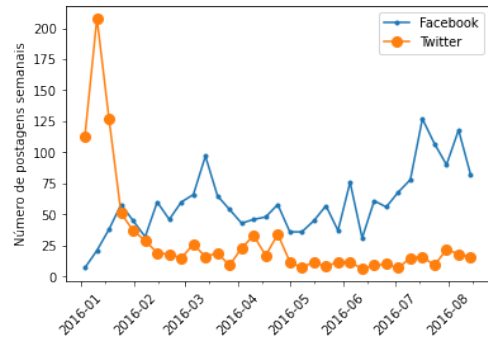

(a) 2016

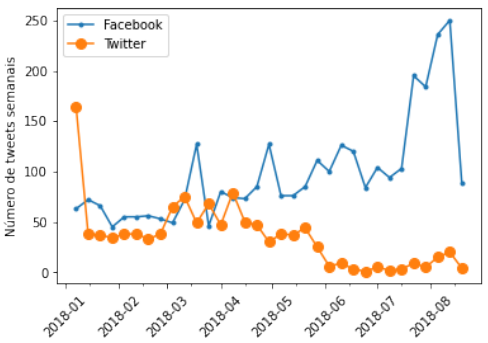

(b) 2018

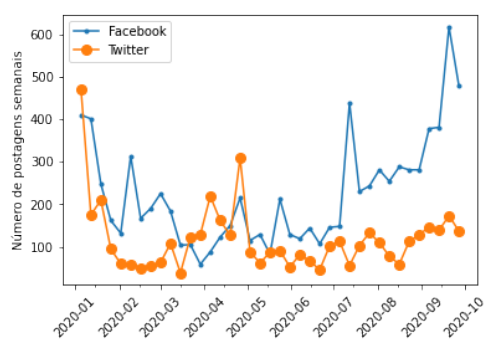

(c) 2020

Figura 1. Número de postagens em cada período pré-eleitoral.

Em seguida, analisamos a distribuição de postagens diárias e semanais nas duas plataformas. No TwITTER-DATASET foram postados diariamente em média 4, 33 tweets em 2016, 5, 10 em 2018 e 17, 17 em 2020. Já no FACEBOOK-DATASET a média de publicações diárias foi de 10, 85 (2016), 14, 43 (2018) e 32, 60 (2020). Analisando semanalmente, o TWITTER-DATASET teve em média 29.84 (2016), 35, 09 (2018) e 118, 43 (2020) tweets. Por fim, FACEBOOK-DATASET teve média semanal de 73.51 publicações em 2016, 99, 27 em 2018 e 225, 71 em 2020. Tal vantagem do Facebook sobre o Twitter se deve ao uso de grupos do Facebook para compartilhar conteúdos de apoio aos candidatos.

\subsection{Narrativas}

Nós selecionamos randomicamente 300 postagens (100 por período eleitoral) do TWITTER-DATASET e FACEBOOK-DATASET para serem rotuladas por 3 voluntários independentes. A rotulação consistiu em classificar cada publicação da amostra como propaganda eleitoral ou não propaganda eleitoral. Para uma maior clareza, nós chamaremos os tweets rotulados de TW-LABELED e as publicações do Facebook de FB-LABELED.

Nós avaliamos a confiabilidade entre os voluntários independentes usando o percentual de concordância e o coeficiente Cohen's Kappa $(\kappa)$ [Oren and Gilbert 2011, Landis and Koch 1977, Savage et al. 2015]. Este coeficiente mede a concordância entre dois voluntários que classificam cada publicação selecionada como uma das duas categorias mutuamente exclusivas. Nós observamos no TW-LABELED que a concordância entre os voluntários, par a par, é de $94,27 \%$ para os voluntários 1 e 2, com $\kappa=0,74$, e 97,30\% para os voluntários 1 e 3 , com $\kappa=0,85$. Para os voluntários 2 e 3, a concordância é de $96,39 \%$, com $\kappa=0,83$. Conforme [Landis and Koch 1977], os valores sugerem uma concordância "substancial". Por outro lado, a concordância entre os avaliadores diminuiu no FB-LABELED, sendo para os voluntários 1 e 2 de $88,55 \%$, com $\kappa=0,58$, e $85,18 \%$ para os voluntários 1 e 3 , com $\kappa=0,51$. Para os voluntários 2 e 3, a concordância é de $86,86 \%$, com $\kappa=0,55$, sugerindo uma concordância "moderada". Especulamos que a maior dificuldade para rotulação das publicações do FB-LABELED esteja associada a complexidade da narrativa no Facebook, que não limita a quantidade de caracteres na publicação, diferentemente do Twitter. 
Com os dados rotulados, pelo menos dois voluntários rotularam $69,02 \%$ dos tweets no TW-LABELED com conteúdo sobre política, e dentro deste percentual, 13,17\% foram rotulados como provável propaganda eleitoral. Além disso, 4,71\% dos tweets estavam relacionados a eleições em outros países, outros 1,01\% eram publicações com elogios a partidos políticos e pré-candidatos, $1,68 \%$ eram ofensas pessoais a políticos, e por fim $27,60 \%$ são de assuntos não relacionados às eleições. No FB-LABELED, $60,94 \%$ das publicações foram rotuladas como conteúdo político ou sobre eleições por pelo menos dois voluntários, sendo 26, 51\% deste percentual possíveis propagandas eleitorais.

Além disso, foram encontrados padrões de narrativas com potencial de propaganda eleitoral antecipada. Porém, é importante destacar que a decisão de enquadrar uma publicação nas redes sociais como propaganda eleitoral é exclusiva dos órgãos fiscalizadores. Dentre as narrativas, destaca-se a indicação de atributos positivos que os eleitores devem buscar nos candidatos, como "Você que é famíliar de militar ou policial, vote no candidato que vai da mais apoio aos militares e policiais. \#CANDIDATO2018". Há também candidatos fazendo pedidos explícitos de votos, mesmo estando completamente fora do período de propaganda eleitoral, como por exemplo tweet: "Meus amigos, eu gostaria de ter o seu voto para Prefeito em 2016. 2016 é um ano de eleição municipal. $[\ldots] "$.

\subsection{Presença de bots}

Nós utilizamos API do Botometer ${ }^{4}$ para verificar se um usuário no TwITTER-DATASET é um bot [Sayyadiharikandeh et al. 2020]. A ferramenta retorna uma probabilidade de um usuário ser bot e também um score entre 0 e 5 para a probabilidade de um bot ser Fake Follower (aumento de seguidores), Self Declared (contas autodeclaradas como bot), Astroturf (bots políticos) e Spammer (spambots). No total, analisamos 2.880 usuários únicos nos anos de 2016, 2018 e 2020.

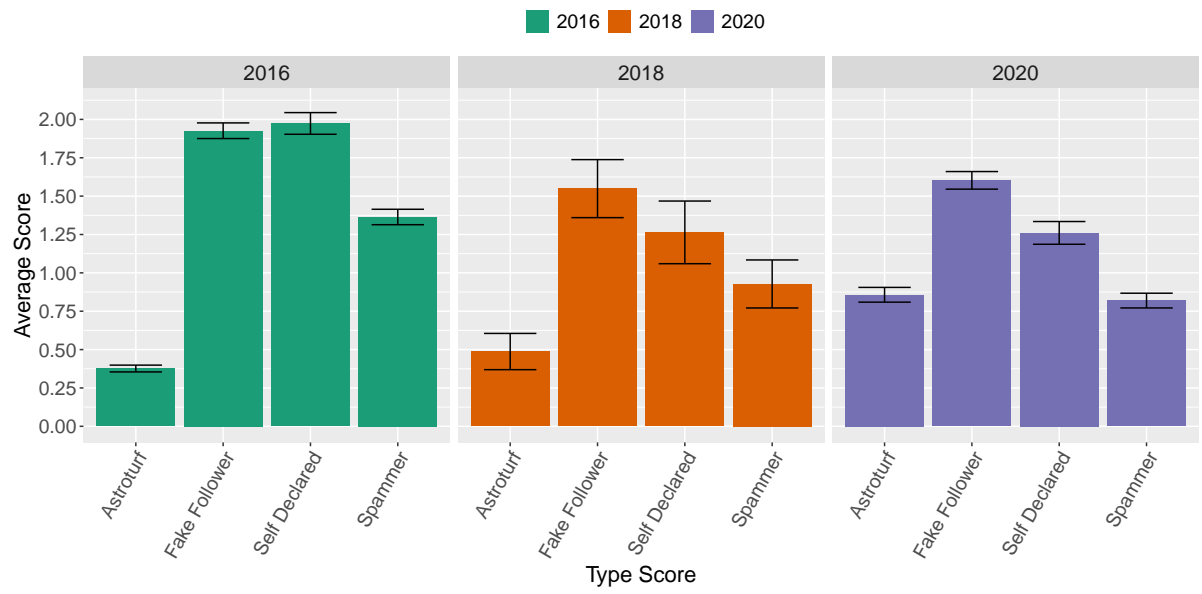

Figura 2. Média de scores Botometer.

A Figura 2 mostra a média de scores para cada tipo de bot. Nós identificamos que cerca de 50\% dos usuários em cada ano $(2016,2018$ e 2020) tem score geral maior ou igual a 0.75 . Isso demonstra que metade dos usuários ativos tem alta probabilidade de

\footnotetext{
${ }^{4}$ https://github.com/IUNetSci/botometer-python
} 
serem bots. Também observou-se que em 2016 e em 2020, anos de eleições municipais, houve maior presença de usuários com probabilidade superior a 0.9. Analisando os diferentes tipos de bots em cada ano, observa-se um aumento gradual da média dos scores de Astroturf e uma diminuição da média de scores de Spammer ao longo do tempo. Logo, de acordo com o score Botometer, em 2020 houve maior presença de bots políticos no Twitter em relação a 2016 e 2018 e menor participação de bots geradores de spam.

\section{Conclusões e Trabalhos Futuros}

A adoção das redes sociais online como ferramenta de comunicação em anos eleitorais é uma grande tendência, pois esses ambientes propiciam uma maneira rápida e eficaz para comunicação dos eleitores com seus candidatos.Todavia, os desafios tecnológicos envolvidos também são grandes para coibir práticas irregulares de divulgação de propaganda política no período pré-eleitoral. Neste cenário, este trabalho apresentou uma metodologia de coleta e análise de possíveis propagandas políticas realizadas no Twitter e Facebook, durante os períodos pré-eleitorais de 2016, 2018 e 2020 no Brasil. Por meio da análise dos dados coletados, evidenciou-se a presença de potenciais propagandas eleitorais antecipadas, o que validou o nosso método de coleta através de uma lista de palavras-chave. Por fim, como trabalhos futuros pretendemos investigar outras redes sociais, bem como explorar o potencial de abordagens automáticas baseadas em aprendizado de máquina para identificação deste tipo de conteúdo.

\section{Referências}

Conover, M., Ratkiewicz, J., Francisco, M., Gonçalves, B., Menczer, F., and Flammini, A. (2011). Political polarization on twitter. In Proc. of the ICWSM.

Grimaldi, D. (2019). Can we analyse political discourse using twitter? evidence from spanish 2019 presidential election. Social Network Analysis and Mining, 9(1):1-9.

Landis, J. R. and Koch, G. G. (1977). The Measurement of Observer Agreement for Categorical Data. Biometrics, 33(1):159.

Oliveira, L. S. D., Vaz-de Melo, P. O. S., Amaral, M. S., and Pinho, J. A. G. (2020). Do politicians talk about politics? assessing online communication patterns of brazilian politicians. Trans. Soc. Comput., 3(4).

Oren, M. A. and Gilbert, S. B. (2011). Framework for measuring social affinity for CSCW software. pages 1387-1392.

Ribeiro, F. N., Saha, K., Babaei, M., Henrique, L., Messias, J., Benevenuto, F., Goga, O., Gummadi, K. P., and Redmiles, E. M. (2019). On microtargeting socially divisive ads: A case study of russia-linked ad campaigns on facebook. In Proc. of the FAT.

Savage, S., Monroy-Hernandez, A., and Acm (2015). Participatory Militias: An Analysis of an Armed Movement's Online Audience.

Sayyadiharikandeh, M., Varol, O., Yang, K.-C., Flammini, A., and Menczer, F. (2020). Detection of novel social bots by ensembles of specialized classifiers.

Silva, M., Oliveira, L. S. d., Andreou, A., Melo, P. O. V. d., Goga, O., and Benevenuto, F. (2020). Facebook ads monitor: An independent auditing system for political ads on facebook. In Proc. of the WWW. 